\title{
FACTORES DEL CAMBIO EN LAS FLUCTUACIONES \\ COYUNTURALES POSTERIORES A LA SEGUNDA \\ GUERRA MUNDIAL
}

Una de las circunstancias más relevantes que registra la historia económica de las últimas décadas, es el cambio profundo que ha experimentado la evolución coyuntural en los países donde predominan los rasgos de la economía de mercado, cambio que se manifiesta, expresado de una manera general, a través de un menor grado de inestabilidad en comparación con la evolución del siglo XIX y las primeras décadas del actual.

Lo que primero llama la atención -en el cuadro de este cambio- es el hecho de que, en los primeros años de esta posguerra ${ }^{1}$ y después, no se han producido crisis y depresiones. En cambio lo que sí se puede observar, son períodos relativamente breves ( de 9 a 12 meses ) de debilitamiento de la actividad económica; llamados en forma bastante generalizada recesiones ${ }^{2}$, que se repiten a intervalos que oscilan entre 2 y 5 años (un promedio de 42 meses) ${ }^{3}$ y cuya intensidad es reducida. El ritmo de la producción industrial

' Contrariamente a los vaticinios de algunos autores como G. HABERLER y Colin CLARCK.

${ }^{2}$ Este término -adaptación de la palabra inglesa, de origen latino (recedere=retroceder), recession- es el que más se utiliza (aparece a veces cuando son muy breves con el prefijo hoy muy en boga en el lenguaje común, "mini"; Cfr. F. DI FENIZIO, "Un momento difficile per gli S. U.", L' industria, año $1967, N^{\circ} 3$, p. 400)

${ }^{3}$ se ha señalado como una característica de estos movimientos que son aperiódicos (Colette CORDEBAS, “¿Es anticuado el concepto de crisis económica?”. Estudios Económicos, Vol. I, $N^{\circ} 1$, enero-junio 1962, p. 101). Estimamos sin embargo que no se trata de un rasgo nuevo ya que también los ciclos conocidos hasta 1929 eran aperiódicos: su "perioricidad" fluctuaba entre 7 y 11 años. W. A. JOEHR considera que la irregularidad de las fluctuaciones coyunturales "pertenece a su esencia más íntima", y que se debe principalmente -sin excluir a otros factores- al carácter "llameante" (la analogia se refiere a la inestabilidad y a los movimientos irregulares de la llama y no al aspecto térmico) del proceso nuclear psicológico social de las fluctuaciones (Cfr. Las fluctuaciones coyunturales, Buenos Aires, Ed. "El Ateneo", 1958, p. 664). 
declina pero en medida mucho menor que durante los retrocesos del pasado. La contracción de la producción manufacturera generalmente no supero el $10 \%$ ( frente, por ejemplo, al $35 \%$ en los retrocesos de $1920 / 21$ y $1937 / 38$ ). El nivel de ocupación experimenta variaciones no estacionales, pero suaves: el desempleo no supera el $7 \%$ (frente al $10 \%$ como mínimo que marcan las depresiones coyunturales del pasado llegando hasta el $24 \%$ y $19 \%$, en la Gran Depresión y el retroceso de 1937/38, respectivamente). En cuanto a los precios y los salarios, son, por regla general, reacios a la baja (de manera especial los últimos) habiendo por el contrario conservado su flexibilidad hacia arriba. Es asimismo notable que en el ingreso se registran más bien disminuciones relativas, y cuando se da el caso raro de disminución absoluta, la misma es muy reducida.

Estos movimientos no se pueden encuadrar en el esquema cuatrifásico de Haberler, que había adoptado últimamente la literatura coyuntural, ni en ningún otro anterior ${ }^{4}$. Y, sin embargo, estamos frente a un movimiento que alcanza un "máximo", llamado también "punto de flexión superior" y desciende a un "mínimo", o punto de flexión inferior, momento que cierra el ciclo $^{5}$ transcurrido y, naturalmente, inicia el ciclo siguiente" ${ }^{6}$. Es pues un movimiento que se compone de dos fases y que -aún en el caso de que no tenga nada que ver con el ciclo económico clásico y cualquiera sea su naturaleza- se presenta, por lo menos hasta ahora, en forma recurrente. Los dos tipos de fases que se observan, no acusan esa "simetría" que se podía observar en el pasado, no en todos pero en la mayoría de los aspectos, entre las fases de prosperidad y las de depresión. Esa "simetría" no se presenta ni siquiera en la duración de las fases. En el ciclo clásico, aún cuando la duración de la prosperidad y la depresión variaba de un ciclo a otro (y más aún en

\footnotetext{
${ }^{4}$ Nos referimos a los esquemas de Jevons, Spiethoff, Wagemann y del Instituto de Harvard. ${ }^{5} \mathrm{Si}$ bien se puede observar en los economistas europeos (en cambio no en los anglosajones) cierta reserva hacia la palabra "ciclo" cuando se trata de fluctuaciones posteriores a la Segunda Guerra Mundial, (Cfr. Erik LUNDBERG, Instability and Economic Growth, New Haven y Londres, Yale University Press, 1968, p. 9), también hay excepciones. Por otra parte hay que tener en consideración que "para muchos economistas 'el ciclo' ha perdido gran parte de su significado" (Cfr. James TOBIN, "The Business Cycle in the post-war world: a review”, Quarterly Journal of Economics, Mayo 1958, p. 284).

${ }^{6} \mathrm{Cfr}$. F. DI FENIZIO, "In vent'anni l'economía italiana ha superato sette periodi di crisi". L'Industria, año 1966, cuad. 1, p. 97.
} 
función de si se trataba de una fase ascendente o descendente del ciclo Kondratieff), el promedio de duración de los períodos de prosperidad no distaba mucho del de los períodos de depresión. En los movimientos actuales, en todos los casos, las recesiones han sido, tal como se ha mencionado, considerablemente más breves que los períodos de expansión. Debemos agregar, por otra parte, como otro rasgo nuevo, la posibilidad latente de una tercera "fase" o sea el peligro de que una fase de expansión, una vez alcanzada la plena ocupación o niveles próximos a la misma, degenere en una inflación.

Estas fluctuaciones no alcanzan a la totalidad de le economía ni a todas las industrias. Se ha producido, en tal sentido, una "desintegración"7 de la coyuntura la que actualmente es más bien "parcial"8 o "semiglobal" cuando no "sectorial". . Las industrias que suelen ser afectadas en primer lugar son las de bienes de consumo, de manera especial duradero. La transmisión a otros sectores no se realiza como antes. Las observaciones formuladas hasta ahora no son concluyentes en cuanto a la vigencia de la aceleración ${ }^{10}$, aunque en líneas generales parece prevalecer la impresión que no funciona como en el pasado. Además no hay procesos acumulativos y las fluctuaciones del estado de ánimo han perdido la intensidad de los tiempos pasados: en ningún momento de las fluctuaciones posteriores a la Segunda Guerra Mundial se han dado estados de optimismo o pesimismo llevados al paroxismo ni escenas de pánico, como los que registró la historia coyuntural hasta la Gran Depresión.

\footnotetext{
${ }^{7}$ Cfr. Rolf FRICKE, Wirtschaftsordnung und Konjunktur, Frankfurt a.M., Ed. Klostermann, 1958 , pp. 108 y 121.

${ }^{8}$ Crf. FRICKE, op. cit., p. 119.

9 Cr. C. CORDEBAS, op. cit., pp. 101, 104. De ahí que las fluctuaciones del producto global y del ingreso nacional sean tan reducidas, lo que hace a CORDEBAS sugerir que la observación de los desajustes se realice sobre la base de las relaciones intersectoriales. (op. cit. p. 105).

${ }^{10}$ Según algunos autores (Cfr. L.A. VINCENT, "La productivité nationale et le mouvement cyclique en France de 1949 a 1963", Etudes et conjuncture, sept. 1964, p. 7; también J. MARCHAL, Expansion et récession, París, Ed. Cujas, 1965, pp. 51 y 55 ) la , misma es evidente. En cambio CORDEBAS (op. cit. p. 104) encuentra que la reacción de las industrias de bienes de capital a las variaciones del consumo es demasiado débil como para poder considerar que tiene todavía vigencia.
} 
Por fin, otra de las características de los movimientos coyunturales del presente, que los distingue del ciclo económico clásico es que, por regla general, los mecanismos de propagación internacional no funcionan como en el pasado ${ }^{11}$. Los períodos de expansión y de recesión de las distintas economías no coinciden, en general, ni están desfasados de manera tal que dejen traslucir en forma clara y evidente tal propagación. No se puede hablar de una insensibilidad total de las economías nacionales a las coyunturas de otros países; incluso se puede afirmar todo lo contrario con respecto a los países integrados económicamente entre sí, donde se nota evidentemente una sensibilidad coyuntural recíproca más pronunciada que antes. Pero si se hace abstracción de estas situaciones la sensibilidad coyuntural de las economías industrializadas es por regla general bastante reducida. Un caso especial constituye la notable disminución, a través de la década del 50, de la sensibilidad coyuntural de las economías europeas occidentales frente a las fluctuaciones del centro coyuntural del bloque occidental, o sea frente a la economía de EE.UU' ${ }^{12}$.

Contrasta con el estado de cosas que acabamos de caracterizar muy brevemente la poca atención que ha merecido hasta el presente por parte de la Ciencia Económica contemporánea ${ }^{13}$ la que -podemos afirmarlo son

\footnotetext{
"Por eso, R. FRICKE habla de una "desintegración" de la coyuntura también a nivel internacional (Cfr. op. cit. p. 111).

${ }^{12} \mathrm{Cfr}$. al respecto la obra de J. MARCZEWSKI, L'Europe dans la conjoncture mondiales, Cahiers de I'Institut de Science Economique Appliquée Supplément, № 136-137, abrilmayo 1963, pp. 39- 60. Marczewski llega incluso a la conclusión que la coyuntura de EEUU ejerce una especie de influencia compensadora sobre la coyuntura europea: deflacionista cuando ambas coyunturas están en expansión, inflacionista cuando ambas están en recesión (Cfr. op. cit. pp. 41-44). Cfr. también V. MARRAMA "E necessaria una revisione de la teoría cíclica?", Rivista internazionale di scienze sociali, vol. XXX, fasc. 1., (enero-febrero 1959), p.17, quien advierte que en 1957/58 la coyuntura norteamericana influyó sobre la europea porque ambas estaban en la misma fase.

${ }^{13}$ Basta, para comprobar esta actitud, un vistazo por los artículos incluidos en Reading in Business Cycles (Allen \& Unwin Ltd., Londres 1966), obra que se puede considerar como continuación de los Ensayos sobre el ciclo económico, publicados en 1944 por Haberler, y que por consiguiente reúne, en cierto modo, los principales aportes que -por lo menos según el criterio del comité de la American Economic Association que tuvo a su cargo la selección- se han publicado en los últimos 25 años. La diferencia que se puede notar en dichos aportes reside más bien -tal como dicen los autores del prefacio (R.A. GORGON y L.R. KLEIN)- en la "reformulación de la teoría de los ciclos económicos en términos
} 
incurrir en ninguna exageración- esté todavía en deuda ${ }^{14}$. ¿Esta actitud puede atribuirse a la gran preocupación por los problemas del desarrollo o, quizás, a un exceso de prudencia por no considerarse todavía suficientemente largo, como campo de observación, el período en cuestión para arribar a conclusiones valederas?.

Por nuestra parte estimamos -tal como ya hemos afirmado en otra oportunidad $^{15}$ - que el problema del desarrollo, lejos de restar importancia al problema coyuntural, la acentúa. En cuanto al campo de observación, un cuarto de siglo constituye un período suficientemente largo como para efectuar por lo menos un balance de los factores a los que se pueden atribuir los cambios. Es esta la tarea que nos proponemos realizar en el presente trabajo: hacer un inventario de todos aquellos factores que, en una forma u otra, han contribuido y/o contribuyen a que el panorama coyuntural en este último

matemáticos y la consiguiente proliferación de modelos dinámicos" así como "la aplicación de las técnicas econométricas en la investigación empírica" (p. V). Con respecto a la realidad coyuntural actual se afirma que (preferimos aquí citar en el idioma original) "We feel that cycles are still with us", mientras que al cambio del panorama coyuntural los autores del prefacio sólo dedican cuatro renglones en que admiten que el mismo "may be different from the cycles before World War II"... "and stabilization policies may be more effective as a result of our improved understanding of the factores making for economic instability" (p. VI). La aparición de la obra ya citada, Instability and Economic Growth, de Erik LUNDBERG, en que se analizan detenidamente las fluctuaciones coyunturales posteriores a 1945 , correspondientes a cinco países, puede quizás considerarse como un cambio en la actitud señalada. No podemos, en cambio, abrir juicio, en este trabajo, sobre dos obras aparecidas recientemente por haber legado a nuestras manos momentos antes de que el material correspondiente a este número fuera entregado a la imprenta. Se trata de: American Business Cycles de C.A. BLYTH (Londres, George Allen and Unwin Ltd., 1969) e Is the business cycle obsolete?, por varios autores, publicado por Martin Bronfenbrenner (J. Wiley \& Sos, Londres 1969), obras que nos proponemos comentar en el próximo número de esta revista.

14 Según J. TOBIN el análisis económico suele estar en atraso con respecto a los acontecimientos reales (Cfr. J. TOBIN, op. cit., p. 287). A. COTTA habla del "eterno atraso" de los intentos de explicación de las fluctuaciones "con respecto a los hechos" (Cfr. Theorie générale du capital, de la croissance et des fluctucions, Dunod, Paris 1967, pp. 217 s.). En este sentido similar se manifiesta también E. LUNDBERG (op. cit., pp. 15 y 38 ).

is Cfr. nuestra nota "La actualidad de los problemas de las fluctuaciones económicas", Estudios Económicos, Vol. III, № 5/6 (enero-diciembre 1964), p. 125. 
cuarto de siglo sea tan distinto del que caracterizó al siglo pasado y las primeras décadas del nuestro.

Conviene precisar que nuestras consideraciones se refieren a los movimientos que experimentan los países que ya han alcanzado un alto nivel de desarrollo ya que es en ellos donde se observaban, en el pasado, en forma genuina, los ciclos económicos y donde por consiguiente también se pueden percibir los cambios en la evolución coyuntural así como factores que los han producido. Esto no significa sin embargo que para los países en desarrollo el problema carezca de interés ${ }^{16}$. Todo lo contrario, estimamos que, justamente en la situación actual, presenta para tales países nuevas facetas de importancia tal, que merece, a nuestro parecer, un tratamiento especial.

Es nuestra convicción que los factores que han contribuido al cambio, aún cuando no todos en la misma medida, son numerosos y de índole muy diversa. Agruparlos en forma sistemática o colocarlos en un orden estricto de importancia es muy difícil. Estimamos, sin embargo, que el primer lugar corresponde al comportamiento de los sujetos económicos y de aquellos participantes de la actividad económica cuyas decisiones imprimen su cuño al proceso económico: las empresas, los sindicatos, los consumidores y - como un ente cuya presencia en la economía se siente actualmente mucha más que en el pasado y que, por su gravitación, trataremos con anterioridad a los demás - el Estado. Corresponde tratar, luego las condiciones tecnológicas. En el tercer lugar trataremos las condiciones económicas internacionales y, por fin, en el cuarto, el aporte con el cual puede haber contribuido al cambio la Ciencia Económica.

\title{
I
}

CAMBIOS EN EL COMPORTAMIENTO DE LOS PARTICIPANTES DEL PROCESO ECONOMICO

\author{
A. El Estado
}

El comportamiento del Estado frente a la actividad económica ha experimentado en las últimas décadas una honda transformación. Ya hace

${ }^{16} \mathrm{Cfr}$. nuestro trabajo citado en la nota anterior, pp. 125 y 126. 
tiempo que ha abandonado la actitud neutral en función de la cual se limitaba a asegurar a los sujetos económicos condiciones favorables para el libre ejercicio de sus actividades. Hacia fines del siglo pasado se producen (haciendo abstracción de las prácticas proteccionistas) sus primeras intervenciones en los asuntos económicos, que Sombart caracterizara como las "primeras canas en la cabellera del capitalismo". La primera guerra mundial y sus consecuencias motivan una acentuación de esta nueva actitud. Pero es recién como reacción a las graves consecuencias de la Gran Depresión, y de manera especial con posterioridad a la Segunda Guerra Mundial, cuando el Estado no se contenta ya ni con esta actitud meramente intervencionista, y asume la responsabilidad de asegurar en forma sistemática la buena marcha de la economía. Esta nueva actitud, que ha sido caracterizada de "fáustica"17 , marca, si aplicamos la periodización de Sombart, la transición definitiva del capitalismo en apogeo al capitalismo tardío.

Pero el cambio de actitud del Estado se manifiesta también en otro aspecto: el Estado no se limita a administrar los bienes que son de su dominio y los fondos públicos provenientes de los impuestos que recauda, sino participa en el proceso económico también como empresario, hecho que en el siglo XIX constituía una excepción y -desde el punto de vista de la doctrina dominante- una aberración. Amplios sectores de la economía considerados de interés público o importantes para fines de la política económica o para la seguridad (como gran parte de los transportes, las comunicaciones, la siderurgia, etc.) están en muchos países explotados actualmente por el Estado. Se trata de otro cambio en los rasgos de la organización económica del sistema, que marca el fin del apogeo del capitalismo: se ha terminado con el dominio indiscutido de la iniciativa privada. Este cambio también se ha manifestado, aunque en menor grado, en países en que las ideas (si bien ya no tanto las prácticas) liberales están aún muy arraigadas.

El cambio de actitud del Estado, en ambos sentidos $-1^{\circ}$ como regulador de la vida económica (esfera gubernamental) y $2^{\circ}$ como empresario (las empresas públicas)- constituye quizás el factor más importante del cambio que se ha producido en la evolución coyuntural.

\section{Función de control y regulación de la economía}

Es el aspecto más conocido en relación con el nuevo panorama

${ }^{17}$ Cfr. J. MARCHAL, op. cit. p. 11. 
coyuntural. En esta función, el Estado tiene en vista fines entre los que se destacan, generalmente tras: altos niveles de ocupación, ritmo adecuado de desarrollo y estabilidad, y trata de alcanzarlos y conciliarlos. Para alcanzar dichos objetivos actúa en dos planos: a) tomando las medidas de política económica que requieren, en función de los fines propuestos, las distintas situaciones concretas y b) estableciendo el marco legal dentro del cual se desarrolla la actividad económica.

a) Desde la década de la Gran Depresión, el Estado se preocupa por evitar que se produzca desocupación en masa. La plena ocupación figura como uno de los objetivos principales en los programas de todos los partidos políticos, cualquiera que sea su ideología. Para poner en práctica esta política los gobiernos disponen de un amplio instrumental que incluye ante todo las bien conocidas medidas de políticas crediticia, fiscal y presupuestaria, y de manera especial del gasto público (sobre todo las obras públicas, "la gran reserva coyuntural"18). Tales medidas han mostrado su eficacia en repetidas oportunidades evitando que, al producirse la recesión, la misma alcance proporciones graves para la economía. Efectos expansivos tiene también la preocupación -también presente, en mayor o menor medida, en todos los gobiernos- de asegurar un crecimiento sostenido y con un ritmo adecuado, de la economía. De manera especial los países industrializados que cuentan con zonas menos desarrolladas ${ }^{19}$, los programas de desarrollo que se proponen hacer desaparecer los desniveles, dan lugar en forma sostenida a cuantiosas inversiones que movilizan gran cantidad de factores de producción.

La estabilidad está protegida contra los movimientos de baja, por las mismas medidas que regulan el nivel de ocupación y el ritmo de desarrollo. Más acuciante, sin embargo, es el peligro de inestabilidad que puede implicar una expansión demasiado acentuada con posibilidades de degenerar en una inflación. Con el fin de evitar tasas de inflación superiores a las consideradas como "aceptables" 20 , se recurre generalmente a medidas fiscales y restricciones crediticias que frenan la expansión. También estas medidas han

\footnotetext{
${ }^{18} \mathrm{Cfr}$. FRICKE, (op. cit., p. 135, donde se hace especial referencia a la construcción de caminos).

${ }^{19}$ Por ejemplo el "Mezzogiorno" en Italia.

${ }^{20}$ Ponemos "aceptables" entre comillas puesto que hay autores que no aceptan ni la tasa de una inflación lenta, o rampante (Cfr. E. KÜNG, "Los sindicatos y la inflación", Estudios Económicos, año I, Nº1, ene-jun. 1962, p. 14 ss.)
} 
mostrado su eficacia y hasta se puede decir que gran parte de las recesiones que se han experimentado con posterioridad a 1945 en EE.UU, Inglaterra, Francia, Alemania, Italia, para citar sólo países, han tenido como factor principal medidas fiscales y crediticias tendientes a "enfriar" la coyuntura. Por otra parte las recesiones provocadas de esta manera no llegan a ser graves ya que, como hemos visto, si la economía tarde en reanimarse, el Estado tiene sus recursos para estimularla. El Estado tiene pues la posibilidad, tomando las medidas que requiere cada situación, de mantener la actividad económica entre límites que sean compatibles con los fines que persigue.

b) El marco legal al que debe ajustarse la actividad económica también ejerce una influencia poderosa sobre la marcha de la economía ${ }^{21}$. Evidentemente este conjunto de normas también se ajusta a los fines de la política económica siendo en tal sentido susceptible de cambios aunque en este caso los mismos suelen producirse, por regla general, a intervalos más bien largos. En realidad en este aspecto la presencia del Estado no es tan nueva ya que la actividad económica siempre se desenvolvía dentro de cierto marco legal. Pero en el capitalismo en apogeo, éste se limitaba a pocos aspectos de los cuales enumeramos los de mayor trascendencia desde el punto de vista coyuntural:

aa. En el cuadro de la legislación laboral, las normas sobre el despido constituyen un freno importante contra la desocupación en masa al igual que las referentes a la salarización un freno contra las fluctuaciones del consumo. Por otra parte, el seguro a la desocupación, que quita medios de pago en la expansión, devolviéndolos en la recesión, puede constituir un estabilizador automático (si los fondos no se utilizan, durante la expansión, para inversiones).

bb. El sistema impositivo en su conjunto así como cada impuesto en parte, puede influenciar en un sentido u otro sobre la coyuntura. De importancia

\footnotetext{
${ }^{21}$ El Estado contribuye así en forma decisiva a la configuración del marco institucional dentro del cual se desarrolla la actividad económica. Estamos conscientes que este no se cubre con el marco legal, siendo más amplio y que, a rigor, correspondería dedicarle una sección aparte. Sin embargo, hemos prescindido de ello por considerar que los aspectos institucionales de mayor gravitación que no caben en el marco legal están tratados en otras secciones de este trabajo.
} 
especial es, sin embargo, el impuesto progresivo a los réditos que constituye otro estabilizador automático, que grava más las empresas en la expansión, aliviándolas en la recesión. Su eficacia depende principalmente de la tasa de progresión, de la eficiencia del sistema impositivo, así como de la proporción que constituyen los impuestos directos en la masa global de impuestos. Si parte de los fondos recaudados en la expansión se destinan a un fondo compensador a utilizarse para obras públicas en la recesión, su eficacia es aûn mayor.

cc. El régimen presupuestario permite que el presupuesto del Estado esté en desequilibrio, lo que abre mayores posibilidades a la política de gasto público. Se trata de otro cambio radical de actitud motivado por la Gran Depresión.

dd. Una atención especial merece el cambio radical producido en el régimen monetario. Este ya no es metálico y de regulación automática sino controlado. Complementariamente, también el sistema bancario es controlado y, pues, responde a la política económica del Estado. De manera que, por un lado, no dependiendo más la cantidad de circulante de las reservas metálicas, no hay desde el punto de vista monetario, ningún obstáculo a la expansión, la que, mientras haya disponibilidad de factores y la demanda sea favorable, en principio puede seguir ${ }^{22}$. Por otro lado esta circunstancia -a la cual hay que agregar la política de plena ocupación de los gobiernos y la acción de los sindicatos- tienen por consecuencia un lento pero seguro movimiento alcista de precios conocido bajo la denominación de inflación rampante, que a su vez, constituye un factor expansivo ya que estimula a las empresas como a los consumidores ${ }^{23}$. Sin embargo, esta situación tiene, también sus aspectos negativos de los cuales el que nos interesa desde el punto de vista coyuntural es la amenaza de que la expansión degenere en una inflación incontrolable. Esta amenaza se puede concretar cuando -de manera especial si el nivel de ocupación alcanza o está próximo al de plena ocupación ${ }^{24}$ - se produce una

\footnotetext{
${ }^{22}$ Esta circunstancia permitiría quizás llegar a la conclusión que, por lo menos en cuanto a la explicación de los puntos superiores de flexión del movimiento cíclico de tipo clásico, Hawtrey tenía razón (Cfr. R.G. HAWTREY, Monetary Reconstruction, $2^{\mathrm{a}}$ ed. Londres, 1926, p. 135).

${ }^{23}$ Así también FRICKE, op. cit., p. 141.

${ }^{24}$ Cfr. MARCHAL, Expansion et récession, p. 104 y ss. Para evitar este peligro, Marchal
} 
rigidez del lado de la oferta de bienes, o si la autoridad monetaria efectúa anticipos al tesoro del Estado, con el fin de cubrir un déficit presupuestario.

ee. El Estado puede modificar la estructura de los mercados e influenciar en la conducta de los oferentes y demandantes.

ff. El Estado puede establecer normas especiales para la explotación de determinados sectores.

gg. Actividades consideradas de interés público pueden ser desarrolladas por el sector público.

hh. El Estado puede establecer normas que fomenten el acercamiento entre los distintos grupos de intereses que actúan en la economía con el fin de allanar diferencias y evitar conflictos que pudieran comprometer la estabilidad económica.

Tanto mediante su acción reguladora como a través del marco legal que establece a la actividad económica -de manera especial en los aspectos bb, ee, ff y gg- el Estado crea diferencias entre los distintos sectores de actividad económica en cuanto a las condiciones en las cuales actúan las empresas respectivas. En tales diferencias podemos encontrar una de las causas principales de lo que se ha llamado la "desintegración" de la coyuntura en coyunturas parciales. También en la acción reguladora del Estado estimamos que podemos encontrar una explicación para las divergencias de opinión con respecto a la vigencia del acelerador: en la medida en que las inversiones obedecen a criterios fijados por la política económica del Estado, dicho principio no encuentra aplicación.

\section{El Estado como empresario}

No persiguiendo fines de lucro, la empresa pública no reacciona a

recomienda la inmigración de mano de obra de otros países (lo que se está aplicando de manera especial en Alemania, donde en 1970 el número de obreros extranjeros ha alcanzado 1.800.000, pero también en Francia, Suiza, Austria) o, del mismo país desde el sector agrícola. Otra solución es la automatización. 
las fluctuaciones del mercado en la misma forma que la empresa privada. Su comportamiento se podría caracterizar, en principio, como a-cíclico, por lo cual, según su dimensión en proporción al sector privado así como según la influencia que ejerce sobre el resto de la economía, constituyen un elemento de estabilidad. Cabe agregar que la empresa pública también puede desempeñar eficazmente, dentro de su sector, funciones al servicio de la política coyuntural del Estado.

Tal como ya se ha mencionado, la participación de la empresa pública en el proceso económico ha aumentado en forma considerable ${ }^{25}$, lo que, sin lugar a dudas, constituye un factor moderador de las fluctuaciones coyunturales.

\section{B. Las empresas}

También en el comportamiento de las empresas se han producido cambios que, si bien menos espectaculares que en el caso del Estado (esta es quizás la causa por la cual no se les acuerda mucha atención), tienen importantes repercusiones sobre la evolución coyuntural. Se trata de cambios que afectan las raíces mismas del espíritu económico capitalista, pero también, y de manera especial, su actitud frente al problema coyuntural. Las causas están, en gran parte, como en el caso del Estado, en las graves repercusiones de la crisis de 1929 y de la Gran Depresión, pero también en otros factores como la evolución de la tecnología así como de la organización y gestión de le empresa.

Ya con anterioridad a la crisis de 1929 se había producido en las sociedades por acciones una evolución en cuanto a la función del empresario, orientada a una separación entre la misma y la propiedad de los medios de producción. Esta escisión no puede dejar de influencia sobre el espíritu de lucro, que, sin desaparecer o ser menor, adquiere otro matiz ${ }^{26}$. Con

\footnotetext{
${ }^{25}$ Estados Unidos, país reacio a la actividad empresarial del Estado, y donde el sector público es más bien reducido, constituye la excepción a esta evolución.

${ }^{26}$ Comparable quizás - mutatis mutandis- con el del jefe de empresa de la economía centralizada (Cfr. Pierre KENDE, "L intéret personnel dans le systême d’économie socialiste", Revue Economique, Vol. X, No3, (may. 1959), pp. 349 ss. y p. 363). Aunque en la economía de dirección central se tiende, por regla general, a maximizar la producción mientras que en la de mercado se tiende a maximizar las ganancias.
} 
posterioridad a la Gran Depresión, y de manera especial después de la Segunda Guerra Mundial se puede observar una nueva interpretación del espíritu racional ${ }^{27}$ : existen tendencias a incluir en el cálculo económico las repercusiones que pueden tener sobre la empresa problemas que a primera vista no la afectan directamente, como, de manera especial, los sociales ${ }^{28}$. Se puede hablar de tendencias, si bien no tan visibles ni generalizadas, a una toma de conciencia en cuanto al rol y la responsabilidad de la empresa en la economía y, en general, en la sociedad ${ }^{29}$. Hay incluso casos de manifiesta actitud solidarista de los empresarios frente a sus obreros ${ }^{30}$, así como situaciones en que los obreros participan en la gestión de la empresa -a veces en forma institucionalizada ${ }^{31}$ - o en las ganancias.

Por otra parte, en forma más específica, se opera un cambio también en cuanto a la actitud y capacidad de reacción del empresario frente a la coyuntura misma. El impacto de la crisis de 1929 y la Gran Depresión dejan como saldo una pronunciada "conciencia coyuntural" y, consecuentemente, una mayor prudencia frente a los vaivenes de la economía ${ }^{32}$. Esta nueva actitud

${ }^{27}$ H. THOMASSEN (Business planning or economic stability, Public Affairs Press, Washington, 1958, p. IV) habla del "carácter autodestructivo de la racionalidad elemental". ${ }^{28}$ LUNDBERG observa que, cuando se trata de despidos, los empresarios proceden con mayor lentitud que en el pasado. La explicación de tal actitud, sin embargo, sería -cuando se espera que la reducción de la demanda sea pasajera- más bien el temor de que, como consecuencia de la política de pleno empleo, la empresa se pueda encontrar en el momento en que mejoren las condiciones en el mercado, en la imposibilidad de reincorporar los obreros despedidos (op. cit., p. 58).

${ }^{29}$ Cfr. THOMASSEN, ibidem.

30 Por ejemplo, en Italia, la empresa Olivetti.

${ }^{31}$ Así la "cogestión" en Alemania. Véase al respecto: Fritz VOIGT, Zur Theorie und Praxis del Mitbestimmung, Schriften des Vereins für Socialpolitik, Neue Folge $n^{\circ} 24 / \mathrm{I}$, Berlin, ed. Duncker \& Humblot, 1962. Los resultados de esta experiencia han sido analizados durante los años 1968 y 1969 por una comisión designada por el Gobierno Federal de Alemania e integrada por el mismo Prof. VOIGT, y que llegó -de acuerdo con lo manifestado por el Prof. Voigt al autor de este trabajo- a conclusiones favorables para la "cogestión calificada".

${ }^{32}$ De manera especial en EEUU (Cfr. THOMASSEN, op. cit., p.1, quien incluso propone una política de estabilidad coyuntural con prescindencia del Estado, fundada sobre la acción-no anticíclica sino "no cíclica", Cfr. p. 30- de la empresa) donde la Gran Depresión ha dejado una especie de "trauma coyuntural". Importante en tal sentido es también (en las economías europeas) el cambio de actitud del empresario frente a la acción del Estado 
se ve reforzada además por la información coyuntural, cada vez más confiable, con que cuenta el empresario. A la misma contribuyen, además de los rápidos medios de comunicación, las reparticiones públicas de estadística pero de manera especial y en forma muy acabada y accesible los institutos de observación coyuntural ${ }^{33}$, así como el uso de las computadoras ${ }^{34}$-en los casos de las grandes empresas ${ }^{35}$ (que, además, cuentan con sus propios asesores económicos y hasta institutos de observación coyuntural)- con el efecto de reducir considerablemente la incertidumbre. Agreguemos, por fin, que la misma escisión a la cual aludimos en el párrafo anterior tiene como consecuencia una profesionalización de la función del jefe de la empresa así como una mejor preparación del mismo, la que en las empresas automatizadas resulta imprescindible ${ }^{36}$.

Estos cambios hacen que el empresario no actúe tan influenciado por sus estados de ánimo o la imitación y que, en general, cometa menos errores. Es decir - hablando en términos de Schumpeter- que ya no actúa tanto en "tropel", rasgo que en el pasado lo hacía crear y acentuar tensiones, las que abrían al camino a la crisis y agravaban la depresión.

También la tendencia de las empresas a autofinanciarse contribuye a una mayor estabilidad, ya que, otorgando mayor solidez financiera a la empresa, la hacen menos vulnerable a las vicisitudes del mercado ${ }^{37}$.

Persisten en cambio nuestras dudas, expresadas también en otra

(Cfr. FRICKE, op. cit., p. 103) cuyo éxito en el campo de la política de estabilización inspira ahora mayor confianza la que, a su vez, tiene por consecuencia un comportamiento anticíclico de los empresarios (Cfr. A. PREDOEHL, Das Ende der Weltwirtschaftskrise, Reinbeck bei Hamburg, Rowohlt, 1963, pp. 46-47).

${ }^{33}$ Así el Instituto de Harvard en EEUU, IFO en Alemania, ISCO en Italia, INSEE en Francia, Oesterreischisches Institut für Wirtschafts forschung en Austria, para dar sólo algunos ejemplos.

${ }^{34}$ Véase al respecto nuestro trabajo, "Implicaciones cíclicas de las nuevas técnicas de producción", en Estudios Económicos, Vol. 1, № 1 (enero-junio, 1962), p. 56.

${ }^{35}$ Pero también las otras tienen la posibilidad de alquilarlas o asociarse para su uso.

${ }^{36} \mathrm{Cfr}$. nuestro trabajo, ya citado, "Implicaciones cíclicas de las nuevas técnicas de producción", p. 52.

${ }^{37}$ Así también Th. PÜTZ: "Problemas y vicisitudes de la política coyuntural", Estudios Económicos, Vol. I, N² 2, (jul.-dic. 1962) p. 209. 
oportunidad ${ }^{38}$, en cuanto a un efecto supuestamente estabilizador de las prácticas no competitivas, a pesar de opiniones autorizadas en tal sentido ${ }^{39}$ : al efecto favorable de la estabilidad de la empresa y sus posibilidades de aumentar los rendimientos, se opone un efecto desfavorable desde el punto de vista coyuntural por la posibilidad que se da en tales formas de mercado, para mantener altos los precios de los productos reduciendo la cantidad produciendo. Para tales prácticas, en cambio, constituyen un freno las tendencias que se observan en la estructura de los costos, debido a la reciente evolución tecnológica en el sentido de una participación cada vez mayor de los costos fijos. Nos referimos de manera especial a la automatización. Tal como señaláramos en otra oportunidad ${ }^{40}$, tales tendencias actúan en dos sentidos. Por un lado acentúan la tendencia, señalada por Schmalenbach ${ }^{41}$, a seguir produciendo aún ante condiciones desfavorables en el mercado, cuando las mismas son estimadas como pasajeras, ya que interrumpir o reducir la producción resultaría mucho más perjudicial. Por el otro lado, lo que es más importante, obliga a las empresas respectivas a fundar sus previsiones en estudios minuciosos (realizados en sus propios departamentos de investigación por equipos de especialistas en distintas ramas) en cuanto a la posible evolución futura del mercado de sus productos, de los factores, etc., establecer sobre esta base planes de inversiones y producción a largo plazo, planes de los cuales no se apartan. Estamos, aquí, estimamos, frente a un factor importante que contribuye a la moderación de las fluctuaciones coyunturales del último cuarto de siglo, ya que las empresas que actúan de la manera expuesta son de grandes dimensiones y de mucha gravitación en el sector privado, constituyendo de esta manera, junto al sector público, un importante elemento

${ }^{38} \mathrm{Cfr}$. nuestro trabajo ya citado "Implicaciones cíclicas de las nuevas técnicas de producción", pp. 54-55.

39) Según Jean MARCHAL (Expansión et récession, París, Cuyas 1965, p. 15) la "molecularidad" actual de la economía constituye un factor de estabilidad. Véase al respecto nuestra reseña de este libro en Estudios Económicos, Vol. IV, No 7-8, (ene.-dic. 1965), p. 133 y ss.

${ }^{40} \mathrm{Cfr}$. nuestro trabajo ya citado "Implicaciones cíclicas de las nuevas técnicas de producción", pp. 48 y ss.

${ }^{41}$ Cfr. E. SCHMALENBACH, "Die Betriebswirtschaftslehre an der Schwele der neuen Wirtschaftsverfassung" en: Zeitschrift für handelswissenschaftliche Forschung, 1928, pp. 241 y ss. Cfr. también -en cuanto a los efectos coyunturales- W.A.JOHR, op. cit., pp. 333 y ss. 
de estabilidad de la economía ${ }^{42}$.

La estructura de costos mencionada contribuye también de otra manera a la estabilidad de la economía: en las empresas automatizadas, la mano de obra adquiere una posición más fuerte y más estable, por los grandes perjuicios que causaría, en caso de huelga, una interrupción del proceso productivo ${ }^{43}$. Evidentemente, por la proporción reducida de la mano de obra en la combinación de factores, en tales empresas, la incidencia de este efecto es más bien limitada.

\section{Los sindicatos}

Los sindicatos constituyen en las economías modernas un factor de mucha gravitación. Esta situación es resultado de una evolución que tiene sus raíces en el siglo pasado cuando, a pesar de todas las adversidades con que se enfrentaron (de manera especial la prohibición legal de las asociaciones profesionales), los obreros se unen al principio en forma clandestina, o en el mejor de los casos tolerada, logrando recién hacia fines del siglo ser aceptados legalmente. Esta evolución culmina con la institucionalización de los sindicatos en la legislación laboral la que -agregada a la escasez relativa de mano de obra que se observa en los países altamente industrializados ${ }^{44}$ - tiende a fortificar su posición. Esta posición les permite, por un lado, actuar como un freno sobre eventuales reducciones del nivel de ocupación: agregada a las normas establecidas en la legislación laboral en materia de despidos, hace muy poco probable, sino imposible, que se produzca una desocupación masiva. Por el otro lado, contribuye en forma muy eficaz, conjuntamente con las disposiciones legales al respecto, a la rigidez de los salarios hacia abajo,

\footnotetext{
${ }^{42}$ Aunque, sin negar eficacia a este factor, E. LUNDBERG, considera que ni hay que exagerar su importancia ya que tal planificación implica "únicamente la proyección de las inversiones y no los compromisos de compras de los bienes de capital, por los cual tales planes pueden ser rápidamente modificados, y no en última instancia bajo el efecto de las fluctuaciones cíclicas" (op. cit., p. 62).

${ }^{43}$ Cfr. nuestro trabajo "Implicaciones cíclicas de las nuevas técnicas de producción", p. 51 y ss.

${ }^{44}$ Crf. León DUPRIEZ "Konjunktur und Sackularentwicklung”, Zeitschrift für gesamte Staatswissenschaft, Vol. 119, cuad. 2 (abr. 1963), p. 295.
} 
ejerciendo, en cambio, en forma latente o efectiva -según las circunstanciasuna presión hacia arriba que constituye la política más popular, común a todos los sindicatos, y que es la principal causa de la flexibilidad unilateral de los salarios. Ambos efectos contribuyen evidentemente a que el consumo se mantenga, con variaciones pequeñàs, en niveles altos. No se puede negar, en verdad, que dichos efectos -y de manera especial la rigidez de los salarios hacia abajo- reducen la capacidad de reacción de las empresas ante las vicisitudes del mercado, aumentando así su vulnerabilidad coyuntural ${ }^{45}$. Estimamos, sin embargo, que fuera de los efectos depresivos que podrían tener la reducción de la ocupación y la baja de los salarios- esa rigidez puede constituir un factor más que, conjuntamente con la incidencia de los costos fijos, contribuye a la tendencia de adoptar técnicas que ahorran mano de obra (las que, a su vez, producen los efectos que hemos tratado en la sección anterior).

También se puede observar una evolución en la actitud de los obreros en el sentido de una menor virulencia en sus manifestaciones como clase antagónica a las otras. Este cambio se puede atribuir muy probablemente a la tendencia ascendente que se puede comprobar en los salarios reales y por lo tanto del nivel de vida con un consiguiente "aburguesamiento" de los obreros $^{46}$. Esta nueva actitud es favorable para el diálogo, el que , a su vez, constituye un marco favorable para una mayor estabilidad coyuntural.

\section{Los consumidores}

La escasa sensibilidad del consumo ante variaciones en el ritmo de crecimiento de una rama o varias ramas de producción se señala como uno de los principales factores de la moderación de las fluctuaciones coyunturales posteriores a la Segunda Guerra Mundial. Esta constancia del consumo es

\footnotetext{
${ }^{45}$ Cfr. FRICKE, op. cit., p. 121. En forma similar se manifiesta también E. LUNDBERG (op. cit., p. 65).

${ }^{46}$ Según FRICKE (op. cit., p. 103) se agregaría además la circunstancia que, de manera especial en los dirigentes sindicales, habría perdido fuerza la creencia en un futuro desenlace socialista de la sociedad actual. Consideramos, sin embargo, que corresponde acordar a esta observación un valor más bien relativo así como circunscribirla a determinados países como p. ej. de manera especial al caso de la República Federal Alemana.
} 
sin ninguna duda atribuible a la rigidez de los salarios a la baja y su flexibilidad al alza, gracias a factores ya tratados, como la acción de los sindicatos y la legislación laboral, siendo muy importante, en el cuadro de este aspecto, el seguro de desocupación. Mientras que estos factores actúan para los ingresos bajos, actúa sobre los ingresos altos -si bien no con los mismos resultados para el consumo- el impuesto progresivo a los réditos.

Pero hay también otros factores que contribuyen a un nivel de consumo alto y sostenido. Así en la medida en que aumenta la participación de los empleados y obreros en la distribución del ingreso, es menor la inestabilidad del consumo, puesto que los ingresos del empresariado (que orientaba en gran medida el consumo durante el capitalismo en apogeo) es muy sensible a la coyuntura ${ }^{47}$. Luego hay que mencionar de madera especial el cambio de actitud que se ha producido en el consumidor con respecto al crédito para consumo. Décadas atrás, contraer deudas significaba algo negativo para la economía familiar, de manera especial en el caso de bienes perecederos y semiduraderos. Esta actitud, que respondía a concepciones tradicionales de carácter moral con respecto a la solidez de la economía familiar, se encuentra hoy totalmente abandonada. Se recurre al crédito de manera especial en momentos de dificultad (como p. ej. situaciones en que el jefe de familia pasa por un período de desocupación), lo que contribuye a moderar los efectos de factores depresivos. Pero también se solicita en momentos en que se cuenta con suficientes ingresos (incluso lo hacen personas que reciben ingresos altos y que podrían fácilmente hacer frente al gasto con sus propios medios ${ }^{48}$ ) con el fin de liberar la parte respectiva del ingreso y poder atender así otros fines (lo que a su vez acentúa el efecto expansivo de este factor). El consumo es, por fin, relativamente alto en determinados productos para el hogar como consecuencia de la mecanización del trabajo doméstico debido, a su vez, al proceso de democratización de la sociedad que se puede observar a partir de la primera guerra mundial ${ }^{49}$, así como, más recientemente, al encarecimiento de la mano de obra. Como resultado de todos estos factores, han surgido nuevas costumbres ${ }^{50}$ en cuanto al consumo, que de esta manera se mantiene, con leves variaciones en niveles altos.

\footnotetext{
${ }^{47}$ Así también R. FRICKE, op. cit., p. 127.

${ }^{48}$ Cfr. ACKLEY, Teoría Macroeconómica, México, Uteba, 1967, p. 35.

${ }^{49}$ Así también R. FRICKE, op. cit., p. 110.

${ }^{50}$ Jean MARCHAL (op. cit., p. 166) se refiere a la "resistencia de los consumidores a la
} 


\section{II}

\section{CONDICIONES TECNOLÓGICAS}

La evolución tecnológica de las últimas décadas difiere mucho en ciertos aspectos, de la que se registró en el siglo XVIII o aún en el siglo pasado. Se trata de cambios que se han ido produciendo en la forma en que se están realizando las investigaciones científicas que dan lugar a los progresos tecnológicos, cambios que se han producido en dos sentidos. La investigación científica por un lado, se ha ido realizando en forma cada vez más sistemática, y, por el otro, está cada vez más orientada por el mercado. Los dos sentidos se condicionan en cierto modo recíprocamente -de manera especial el segundo al primero- $y$, por consiguiente, lo mismo sucede con sus efectos coyunturales. Se puede afirmar que la circunstancia que las investigaciones científicas en que tienen origen los progresos tecnológicos, se realizan en forma más sistemática, hace que los mismos sean menos resultado del azar y que sea, pues, más frecuentes: lo que evidentemente tiene una incidencia favorable sobre la frecuencia con que se producen las innovaciones (en sentido schumpeteriano), y sobre la marcha de la coyuntura ${ }^{51}$. En cambio, la investigación orientada por el mercado tiene efectos más complejos. Tiene el efecto de concentrar la investigación cobre objetivos determinados, por un lado, por el otro, el de poner al servicio de la misma, gran cantidad de recursos especialmente privados ${ }^{52}$, contribuyendo, de las dos maneras, a una mayor intensidad de las investigaciones y, por esta vía, nuevamente a una mayor frecuencia de los progresos científicos y de ahí de los tecnologicos, estimulando la coyuntura. Pero al mismo tiempo tiene por efecto que los inventos -siempre usando los conceptos de Schumpeter- se transformen con

degradación de su nivel de vida", lo que se manifiesta "con mayor fuerza cuando una contracción tiende a acentuarse, puesto que afecta más profundamente sus costumbres" (p. 167). La eficacia de este freno depende del nivel de ahorro, de ahí que "reaccionará con más fuerza en la medida en que la sociedad en consideración sea rica" (p. 167).

${ }^{51}$ Así también C. CORDEBAS, op. cit., p. 102; y Th. PÜTZ, op. cit., 209.

${ }^{52} \mathrm{Cfr}$. al respecto Yale BROZEN: "Determinants of the Direction of Technological Change", American Economic Review, Vol. XLIII N ${ }^{\circ} 2$, Papers and proceedings of the 65 Annual Meeting of the A.E.A. p. 298. También nuestro trabajo "Implicaciones cíclicas de las nuevas técnicas de producción”, Estudios Económicos, Vol. 1, Nº 1 (ene.-jun. 1962) pp. 40 y ss. 
mayor probabilidad en innovaciones ya que la investigación responde a los intereses de las empresas que la financian. Por fin, no se puede descartar la posibilidad de que la orientación de las investigaciones al mercado, tenga un efecto estabilizador por el hecho de que -respondiendo dicha orientación a situaciones de escasez de determinados productos o factores- las innovaciones resultantes tienden a aliviar las tensiones que podrían producirse por tales situaciones y evitar que las mismas impriman un curso desfavorable a la coyuntura.

Hay que agregar a estos efectos directos, los efectos indirectos de la evolución tecnológica reciente, de manera especial los -ya mencionados- que se producen a través de la estructura de los costos y dimensión de la empresa, así como a través de la mejor información que brindan a la empresa los medios de comunicación moderna y las computadoras, a los fines de una gestión eficaz.

\section{III}

\section{CONDICIONES ECONOMICAS INTERNACIONALES}

El cambio de actitud del Estado se extiende también sobre las relaciones económicas internacionales, donde las medidas de política económica van mucho más allá de las prácticas arancelarias, conocidas también en el capitalismo en apogeo y que actualmente se aplican, evidentemente, en mayor medida. Una gama de técnicas, desarrolladas durante la década del 30 -y de las cuales destacamos las medidas cambiarias- se suma a aquéllas y constituyen todo un instrumental ${ }^{53}$ que permite que los efectos de las recesiones que se producen en el exterior puedan ser asimilados o por lo menos atenuados por medidas de política coyuntural. Evidentemente la capacidad de hacerlo está -también ahora, como en el pasado- condicionada por la relación entre la renta nacional externa y la renta nacional total, el grado de diversificación de la producción, la elasticidad de precios de los productos de exportación en los países que los importan, la elasticidad de

\footnotetext{
${ }^{53} \mathrm{~A}$ lo cual hay que agregar que las mismas medidas de política económica en orden interno pueden ayudar a prevenir o atenuar los efectos de movimientos del exterior.
} 
ingreso de esa demanda en los mismos países. En tal sentido podemos también ahora distinguir varios grados de sensibilidad, cuyos extremos son el caso del país centro, por un lado, y el de los países periféricos por el otro. Pero se han producido algunas situaciones nuevas que no dejan de tener sus consecuencias.

En primer lugar hay que considerar algunas circunstancias que hacen al comportamiento del país que constituye desde la segunda guerra mundial, el centro coyuntural de la economía mundial (con exclusión del bloque colectivista), o sea EE.UU.. Se pueden notar primeramente, cambios en las importaciones estadounidenses. Dichas importaciones, consideradas en forma global, "se comportan de una manera perfectamente cíclica" 54 , pero la intensidad de sus fluctuaciones varía según la procedencia de las importaciones. Las importaciones procedentes de los países de la O.E.C.E. más importantes que antes (la participación en las mismas de los productos manufacturados, de $17 \%$ en 1947 había llegado a 36\% en 1960)- son relativamente mucho menos sensibles que antes de la coyuntura norteamericana ${ }^{55}$, lo que se debe a la mayor estabilidad de los ingresos de que disponen las economías de consumo de EE.UU., protegidas principalmente por la política anticíclica gubernamental así como por los estabilizadores automáticos y la acción de los sindicatos. En cambio son más sensibles que antes las importaciones procedentes de otros países, entre los cuales están incluidos de manera especial los países en desarrollo. Evidentemente, dicha circunstancia contribuye a la relativa insensibilidad ya señalada- de las economías europeas occidentales a la coyuntura norteamericana (así como a una mayor sensibilidad en sentido inverso), constituyendo, en cambio, un elemento más de inestabilidad para las economías en desarrollo ${ }^{56}$. A este efecto que tienen las tendencias recientes de las importaciones del centro coyuntural occidental, hay que agregar el que surge por la actitud de los inversionistas norteamericanos, caracterizada por

\footnotetext{
${ }^{54}$ J. MARCZEWSKI, op. cit., p. 40.

55 "Las exportaciones europeas hacia EEUU siguen siendo sensibles a la coyuntura americana, pero las fluctuaciones que resultan son actualmente demasiado débiles como para afectar seriamente la coyuntura europea". J. MARCZEWSKI, op. cit., p. 41.

${ }^{56}$ Véase con respecto a las economías latinoamericanas, David L. GROVE, Las fluctuaciones económicas en Estados Unidos y América Latina, México, C.E.M.L.A, 1959.
} 
una pronunciada preferencia para los países europeos occidentales. Esta actitud "contribuye a acelerar el crecimiento de las economías europeas y disminuir aún más su sensibilidad a las fluctuaciones de la economía norteamericana interna" 57 (aunque aumenta su dependencia potencial para el caso de una retirada repentina de los capitales norteamericanos), restando en cambio, de esta manera, posibilidades a los países en desarrollo.

También surgen consecuencias de índole coyuntural del rol conductor asumido por EE.UU. en el llamado bloque occidental, con posterioridad a la segunda guerra mundial. Esta posición obliga a EE.UU. a efectuar en el exterior gastos e inversiones militares que, también en este caso, se orientan con preferencia hacia Europa Occidental, lo que acentúa los efectos ya mencionados, favorables para las economías europeas (así como también el negativo, que se suma a la mencionada dependencia potencial, en el sentido de una dependencia potencial de las decisiones políticas del gobierno de EE.UU.). Un efecto favorable constituye, evidentemente, la ayuda al exterior, no militar. Está aquí incluida la ayuda del Plan Marshall, cuyos efectos de recuperación y consolidación de las economías europeas occidentales son bien conocidos. Planes de ayuda de la misma envergadura, sin embargo, no se han producido con posterioridad, por lo cual no contamos actualmente, en este aspecto, con un efecto estabilizador o expansivo que valga la pena mencionar. Por fin, hay que considerar el compromiso que surge de la posición actual de EE.UU., en cuanto a la carrera tecnológica en que se encuentra con respecto a la potencia conductora del otro bloque, la URSS. Dicha carrera ocasiona a la economía norteamericana esfuerzos muy grandes que son sólo posibles mediante una inflación controlada, cuyos efectos expansivos no se limitan a la economía estadounidense sino también se reflejan -se sostiene ${ }^{58}$. sobre las demás economías industrializadas occidentales.

Una evolución que ha tenido importantes efectos en cuanto a la propagación internacional de los movimientos coyunturales, está dada por los movimientos de integración económica, de manera especial por los que se han producido entre las economías europeas occidentales. La circunstancia que los intercambios entre los países económicamente integrados son muy

${ }^{57}$ Cfr. MARCZEWSKI, op. cit., p. 46.

${ }^{58}$ Cfr. FRICKE, op. cit., pp. 140-141. 
intensos ${ }^{59}$, la política coyuntural, en cambio, sigue siendo independiente de un país a otro ${ }^{60}$, produce un doble resultado, llamado por Marczewski "efecto de resonancia" 61 . Por una parte tiende a ampliar las repercusiones o sea propagarlas a todo el grupo, cuando ante una determinada situación coyuntural desfavorable o peligrosa en cualquiera de sus miembros, los mismos se resignan en una actitud pasiva. Por otra, ejerce una acción amortiguadora para todo el grupo, cuando alguno de sus países le sale al paso a la emergencia con medidas coyunturales. Este efecto hace que la sensibilidad coyuntural de los países integrados dependa de la actitud de sus gobiernos, la que es -como hemos visto- propensa a la intervención, por lo cual el efecto final será, son preponderancia, de mayor insensibilidad a la coyuntura exterior al grupo integrado.

Es difícil establecer la medida en que la demanda de bienes de producción generada por los países en desarrollo ha contribuido a la relativa estabilidad coyuntural que se nota, en las últimas décadas, en los países industrializados. Pero es indudable que la toma de conciencia en cuanto a los desniveles de desarrollo -una de las características de toda esta postguerratanto a nivel nacional (a través de políticas o planes de desarrollo) como internacional (a través de la acción de organizaciones internacionales, tendientes a asistir en esas políticas a los países respectivos), ha dado lugar en los mercados internacionales a una demanda que redunda en beneficio de

${ }^{59}$ De manera especial en el caso de la Comunidad Económica Europea, dentro de la cual los cambios responden a un doble criterio: $1^{\circ}$ Ios intercambios de cada país del grupo con los otros países del grupo son superiores a los intercambios con cualquier otro país o grupo de países; $2^{\circ}$ los intercambios del grupo con cada uno de sus miembros son superiores a los intercambios del grupo con cualquier otro país que satisface la condición $1^{\mathrm{a}}(\mathrm{Cfr}$. J. MARCZEWSKI, op. cit., p. 54).

${ }^{60}$ Con respecto a la necesidad, así como bases para un "código internacional" para la coordinación de las políticas coyunturales nacionales, consúltese: Alfred MÜLLERARMACK, "Gedanken zu einem Kodex des richtigen konjunkturpolitischen Verhaltens", Wirtschaftspolitische Chronik, Instituto de Política Económica de la Univ. de Colonia, 1961, cuad. 1, pp. 9-16, así como por el mismo autor: "Konzeption für eine neue Europapolitik", Wirtschaftspolitische Chronik, 1969, cuad. 2/3, pp. 13-18. Sobre los esfuerzos de coordinación de las políticas coyunturales realizados en el seno de C.E.E. consúltese: Jacques y Colette NEME, Economie européenne, París, P.U.F., 1970, pp. 306322.

${ }^{61}$ J. MARCZEWSKI, op. cit., p. 55. 
los países altamente industrializados. Esta demanda, si bien a veces sujeta a problemas de inestabilidad política, tiene un carácter más bien sostenido, por la circunstancia de que, en la misma forma como los países industrializados no falta en ningún programa político el objetivo de plena ocupación, cualquier gobierno de los países subdesarrollados o en vías de desarrollo proclama en mayor o menor medida, el desarrollo como meta principal. Además, sucede a menudo -como se ha podido observar en el caso de las economías latinoamericanas en relación con la coyuntura de EE.UU. ${ }^{62}$ - que las importaciones de tales países reaccionen, ante reducciones de sus exportaciones (por algún movimiento coyuntural de recesión en el país industrial importador de sus productos), con cierto atraso, el que evidentemente actúa de manera atenuante sobre la recesión en el país industrial (agravando al mismo tiempo los efectos de la recesión sobre el país en desarrollo).

\section{IV}

\section{PROGRESOS REALIZADOS POR LA CIENCIA ECONOMICA}

Las investigaciones realizadas con posterioridad a la Crisis Mundial de 1929 enriquecieron la Ciencia Económica considerablemente. Uno de los aspectos en que más se nota este progreso es el coyuntural. El gran salto en este aspecto, se produce -evidentemente bajo los apremios de la gran depresión- de manera especial durante la década del $30^{63}$, pero también en parte durante la guerra así como en los primeros años de esta posguerra, después de lo cual se produce un estancamiento. El resultado es "una mejor comprensión de los factores que contribuyen a la inestabilidad económica", la que, a su vez contribuye a "que las medidas de política de estabilización sean más eficaces" ${ }^{\prime 64}$.

${ }^{62}$ Cfr. David GROVE, op. cit., p. 27 y ss.

${ }^{63}$ Se puede afirmar tranquilamente que la bibliografía que constituye el producto de las investigaciones realizadas durante esa década, supera en mucho todo lo publicado, sobre el tema, con anterioridad a 1920.

${ }^{64}$ Cfr. R.A. GORDON y L.R. KLEIN, Prefacio a la obra Readings in Business Cycles, Londres, Allen \& Unwin, 1960, pp. VI. 
El aporte principal de la teoría económica en tal sentido reside en el enfoque macroeconómico. En realidad este enfoque no constituye nada nuevo ya que tiene sus precursores en los intentos de Quesnay y Marx ${ }^{65}$. Pero tales intentos no podían tener eco ni ser aplicados mientras la doctrina predominante los consideraba innecesarios $-\mathrm{y}$, en cierta forma, hasta peligrosos- ya que, según sostenía dicha doctrina, la mejor manera de asegurar la buena marcha de la economía era no interferir en ella. En tal sentido, se puede decir que el cambio que -bajo los apremios de la Gran Depresión- se opera en la actitud del Estado, abre el camino también al instrumental de análisis macroeconómico que elabora Keynes. El conocimiento de las relaciones que existen entre las principales magnitudes globales -consumo, ahorro, inversióny de las influencias que las mismas ejercen sobre el nivel de ocupación y el ingreso, constituye una gran conquista en la lucha contra las vicisitudes coyunturales.

También en las técnicas de la observación económica se han realizado grandes progresos. Hay que mencionar, en este sentido, en primer lugar, el análisis de insumo-producto (que, por otra parte, está condicionado por los progresos ya mencionados, realizados en el campo de la teoría macroeconómica) cuyo valor reside de manera especial en la posibilidad que ofrece de tener un conocimiento cabal de las relaciones y la interacción entre los distintos sectores de la economía. También en el campo de la observación coyuntural (barómetros económicos) se han realizado progresos. En ambos casos, la eficacia de las técnicas respectivas, ha aumentado considerablemente desde el campo tecnológico, por los progresos realizados en el campo de la electrónica. Las medidas de política económica del Estado, pero también las decisiones de los sujetos económicos, se fundan de esta manera en un conocimiento más sólido y más completo de la realidad de la economía y de la situación coyuntural de un determinado momento ${ }^{66}$.

$* * *$

${ }^{65}$ Véase al respecto nuestro trabajo: "Tres contribuciones a la Teoría del Circuito Económico", Técnica y Economía, Bahía Blanca, No 12 (jul.- dic., 1952).

${ }^{66}$ LUNDBERG se refiere a la "revolución estadística" ocasionada por la Segunda Guerra Mundial. Considera, además, muy importante el hecho que "tanto por parte de los ciudadanos como por parte de los políticos y los economistas, la observación estadística se toma muy en serio" (op. cit., p. 16). Ella se ha transformado en algo así como "nuestro 
Del panorama que acabamos de presentar surgen algunos interrogantes. En primer lugar, ¿responde la teoría coyuntural dominante a la nueva situación? Recordemos en tal sentido que la aceleración, uno de los pilares de la teoría coyuntural moderna, actúa, con limitaciones: no funciona en los sectores nacionalizados ni en los intervenidos, ni tampoco en aquellos sectores privados no intervenidos en que las inversiones se planifican a largo plazo. Esta circunstancia, evidentemente, reduce en forma considerable la aplicabilidad de los modelos que incluyen la aceleración entre sus elementos básicos. También las previsiones de los empresarios, si bien siguen constituyendo un factor importante, están sujetas en medida considerablemente menor a los efectos de los estados de ánimo especiales. En tal sentido, las teorías psicológicas, que en nuestra concepción ocupan un lugar de prioridad ${ }^{67}$ para la explicación de las fluctuaciones coyunturales del pasado, tendrán posiblemente menos actualidad. En cuanto al factor monetario -un tanto descuidado en la teoría coyuntural dominante ${ }^{68}$-vuelve a cobrar un lugar de relieve pero de una manera distinta: a través de las condiciones que abren las compuertas a la presión inflacionaria por un lado, y, por el otro, como instrumento de política coyuntural del Estado. Hay luego, que incluir como factores nuevos al Estado ${ }^{69}$ y a los sindicatos. Todo esto nos lleva a la conclusión que es necesario someter la teoría coyuntural a una revisión ${ }^{70}$,

sexto sentido" (ibid. p. 17). Advierte, sin embargo, sobre los peligros que implica el tomar el mundo estadístico demasiado en serio" (ibid. p. 16, subrayado en el original). En general, hace en varias oportunidades advertencias contra las "pretensiones exageradas de la precisión cuantitativa" (ibid. p. 18).

${ }^{67}$ Entre las mismas destacamos de manera especial, como muy concluyente y completa en cuanto a los aspectos psicológicos que abarca, la teoría psicológicosocial de W.A. JOEHR (op. cit., pp. 620-650).

${ }^{68} \mathrm{Cfr}$. V. MARRAMA, op. cit., pp. 13 y 22. También, aunque en un sentido algo distinto G. HABERLER, "Monetary and Real Factors Affecting Economic Stability: A critique of certain tendencies in Modern Economic Theory" en: Reading in Business Cycle, Londres, 1966, Allen \& Unwin, pp. 130 y 148 y ss.

69 "Los ciclos económicos observados (posteriores a la Segunda Guerra Mundial) no pueden ser comprendidos sin tener en consideración las políticas gubernamentales de estabilización: monetarias, fiscales y otras" (Cfr. LUNDBERG, op. cit., o. 19). Es, por otra parte, necesario advertir sobre "la dificultad de aislar un "mecanismo coyuntural" cambiante-que en cierto modo encierra las reacciones internas de la economía- de los impulsos originados en forma directa por los cambios de la política económica" (Cfr. LUNDBERG, loc. cit.).

${ }^{70} \mathrm{~A}$ igual conclusión ha llegado unos años atrás, V. MARRAMA, op. cit., p. 10 y ss. y, unos años más tarde, si bien no en los mismos términos, J. MARCHAL (op. cit., p. 16). 
con el fin de tratar de elaborar una teoría nueva que responda a las nuevas condiciones. Es una tarea ardua que, estimamos, difícil pueda ser llevada a cabo con éxito mediante aportes aislados y sin una amplia discusión ${ }^{71}$, y que, de todos modos, excede los propósitos de este trabajo. Lo que, en cambio, trataremos de trazar a muy grandes rasgos es un simple esbozo de interpretación sin ninguna pretensión de generalización.

En la multitud de factores que contribuyen al estado actual de cosas, se pueden distinguir, con cierto grado de esquematización, tres grupos. Encontramos, primero, factores generadores de ciclos económicos del tipo clásico que, en mayor o menor medida, tienen todavía vigencia. Un segundo grupo está formado por factores nuevos que actúan en forma expansiva y que son aquellos que dan lugar al peligro de latente de inflación. Por fin el tercer grupo está compuesto también de factores nuevos, pero que actúan como freno tanto en la expansión como en la recesión. Aquí se destaca, actuando sobre los puntos de flexión, la acción del Estado. Esta acción está determinada por decisiones que oscilan, en función de la situación económica, entre dos fines (o grupos de fines): plena ocupación ( $\mathrm{y}$ crecimiento), por un lado y estabilidad, por el otro. Hay como se ha expuesto, una serie de factores

${ }^{71}$ El problema del ciclo económico característico del capitalismo en apogeo ha constituido la preocupación principal de toda una generación de economistas. Llama, en cambio, la atención, la escasa resonancia que tienen pasos importantes hacia una teoría de las fluctuaciones coyunturales acorde con las condiciones económicas actuales, como los emprendidos por V. MARRAMA (op. cit.), J. MARCHAL (op. cit.), así como -si bien no en busca de una teoría de las recurrencias sino de la coyuntura (incluyendo crecimiento y movimientos accidentales)- por J. MARCZEWSKI (op. cit., precedida por La conjoncture économique des Etats-Unis, 1950-1960, Cahiers de M.S.E.A, No 117, sept. 1961) y, más recientemente, por A. COTTA (op. cit.). En cambio, "tenemos ya, aparentemente, un surtido completamente suficiente de bibliografía en la cual los modelos teóricos viven una complicada vida propia un tanto despreocupados por la realidad económica" (E. LUNDBERG, op. cit., p. 14). Esta desconexión entre teoría y realidad tiene, según LUNDBERG, dos causas. "Por un lado, la evolución de la teoría sigue en gran medida sus propias leyes de desarrollo: Las formulaciones y los teoremas nuevos se elaboraron sobre las bases de versiones anteriores y como resultado de esfuerzos tendientes a ampliar los alcances y aumentar la profundidad de los modelos teóricos existentes. Por el otro lado, como economistas, tenemos, al mismo tiempo, una tendencia conservadora en el sentido de seguir apegados a los instrumentos teóricos que hemos aprendido a manejar, y aplicarlos a problemas que están cambiando permanentemente" (op. cit., p. 15). 


\section{ESTUDIOS ECONOMICOS}

(pertenecientes al segundo grupo) que tienen como consecuencia tendencias expansionistas que pueden degenerar en inflación. Cuando este peligro es inminente, el Estado actúa frenando la expansión ${ }^{72}$. Pero "es difícil dosificar las medidas restrictivas. Cuando las mismas son indebidamente severas o se producen fuera de tiempo, se crean las condiciones para la inflexión del movimiento"73. Se produce la recesión, "de la cual la economía sale apenas hayan sido levantadas las medidas restrictivas" "74. Se ha visto, que hay factores que impiden que se desencadene la crisis o que la recesión degenere en depresión. Además, si una vez levantadas las restricciones, la recuperación no se produce en forma espontánea, el Estado pone en acción sus resortes tendientes a poner en marcha la nueva expansión, no se excluye la posibilidad de que los puntos de flexión sean causados por algún otro factor, exógeno o endógeno. Pero hasta ahora el Estado ha tenido en los mismos participación decisiva y todo hace pensar que seguirá teniéndola.

Otro interrogante que se plantea es, si estas fluctuaciones son, siguiendo un lenguaje tradicional, de naturaleza "cíclica" o -empleando una expresión que preferimos incluso para las fluctuaciones coyunturales del capitalismo en apogeo, las que distaban mucho de tener la regularidad que sugiere la palabra "ciclo", prestada de la Física- si son recurrentes. La contestación a esta pregunta depende de la naturaleza de los factores que los producen. Según hemos podido ver, entre estos factores se destaca la participación del Estado, de manera especial en los puntos de flexión. Esto significa atribuir las fluctuaciones en gran parte -si no principalmente- a un origen exógeno ${ }^{75}$. ¿Equivaldría esto a negarles su carácter recurrente?. De todos modos hay que excluir, ante tal constación la hipótesis de que se trata

\footnotetext{
${ }^{72}$ En lo que respecta al rol del Estado en las fluctuaciones coyunturales del último cuarto de siglo, seguimos en líneas generales la explicación de aportada por V. MARRAMA (op. cit., pp. 19 ss.), explicación que consideramos adecuada a las condiciones actuales y, en consecuencia, hacemos nuestra.

${ }^{73}$ Cfr. MARRAMA, op. cit., p. 21.

${ }^{74}$ Loc. cit.

${ }^{75}$ Aunque no sería absurdo poner en duda tal carácter cuando la acción está determinada por el mismo movimiento coyuntural (Cfr. PREDOEHL, op. cit., p. 39, asimismo, nuestra opinión al respecto en nuestra reseña del libro, Estudios Económicos, Vol. II, No 3 (ene.jun. 1963 p. 98).
} 
de fluctuaciones del tipo de Kitchin ${ }^{76}$. Pero no hay que perder de vista el carácter endógeno de otros factores que actúan, así como las condiciones en que actúa el Estado. Este tiene la economía bajo control, pero sus resortes -tanto los "automáticos" como los que dependen de actos de decisión-entran en acción recién cuando las condiciones de la economía lo requieren. O sea, en la medida en que los factores que constituyen un peligro de inflación desenfrenada, sean permanentes, habrá que contar con la acción moderadora del Estado. Y por otro lado, una vez pasado el peligro, habrá que contar con su acción estimulante ${ }^{77}$. Es entonces ocioso hacer especulaciones sobre el carácter exógeno de la acción del Estado, para determinar el carácter recurrente de las fluctuaciones, mientras se de por supuesta una actitud constante del Estado frente a situaciones que -a su vez- tienen por origen factores permanentes. Evidentemente la permanencia de estos factores es relativa, así como históricamente, ha sido relativa la permanencia de las condiciones que generaban las fluctuaciones coyunturales caracterizadas como cíclicas, del capitalismo en apogeo. Indudablemente, no se excluye que la actitud del Estado pueda ser inflúda también por otros objetivos que el de la buena

\footnotetext{
${ }^{76}$ Fernando DI FENIZIO ("Sviluppo e cicli brevi in Italia fra il 1950 e 1957 ", L industria, 1957, No 3 jul.-sept., p. 569), habla de "los ciclos breves o ciclos Kitchin, posteriores a 1945”. Tal hipótesis es sugerida también por Leon BUQUET Orientaciones actuales de la economía, Bs. Aires, ed. Troquel, 1961. También A. PREDOEHL (op. cit., p. 48) se refiere en tal sentido, aunque con suma prudencia ("queremos sólo aludir a tal posibilidad") por considerar el período observado todavía demasiado corto.

${ }^{77}$ Hay que señalar otro aspecto que -en la medida en que reconocemos al Estado un rol preponderante-constituye una diferencia más entre estos movimientos y las fluctuaciones coyunturales del tipo ciclo económico clásico. Las decisiones del Estado están sujetas -en la medida en que se toman en función de la buena marcha de la economía- a la elección entre fines que, aun cuando no constituyen dicotomías, constituyen, en la medida en que es necesario establecer un orden de prioridad, fines alternativos. Por un lado tenemos la plena ocupación y el crecimiento; por el otro la estabilidad. En tal sentido estos movimientos, salvando las diferencias de sistema, se acercan a aquellas fluctuaciones, señaladas como posibles en la economía de dirección central, que surgen como consecuencia de tensiones entre fines alternativos. (Cfr. J.H.G. OLIVERA, "Economic Growth under Collectivism" KYKLOS, Vol. XIII, 1960, cuad. II; J. GOLDMAN "Fluctuations and Trend in the Rate of Economic Growth of some Socialist Countries", Economic Planning, Vol. 4, No 2, Oslo, 1964; y también nuestro trabajo "Contribuciones para una teoría de las fluctuaciones de la economía centralizada", Estudios Económicos, VOL. V, N $9 / 10$, ene-di. 1966).
} 
marcha de la economía (como p. Ej.: fines políticos nacionales o de carácter partidista $)^{78}$ pero se puede suponer razonablemente que el Estado lo tendrá muy en cuenta.

Estimamos, pues, en cuanto a las perspectivas para el futuro, que, mientras perduren las condiciones actuales, se puede hablar de movimientos recurrentes. Hay que considerar además como muy difícil, si no imposible, que se produzcan, en el cuadro de los mismos, crisis y depresiones coyunturales. No se puede en cambio excluir la posibilidad de que se produzcan crisis por causas exógenas, o por los canales de las relaciones económicas exteriores (cambios profundas en los mercados internacionales de bienes, problemas graves en el mercado de divisas, huida repentina y masiva de capitales), a condición de que las alteraciones provocadas en la economía sean de proporciones tales que no puedan ser absorbidas por ninguno de los factores considerados en este trabajo, ni siquiera por la política coyuntural ${ }^{79}$.

Más difícil -si no imposible- es, dada la complejidad de los factores intervinientes, hacer previsiones en cuanto a la permanencia de las condiciones actuales. Las condiciones históricas en que se desarrollan las sociedades son cambiantes y lo son en mayor medida en las épocas recientes. Es, pues, posible que, a corto o largo plazo, se produzcan cambios en dichas condiciones. ¿Reforzarán los mismos la situación actual o la cambiarán? Y, en esta segundo caso, ¿en qué sentido? Si esquematizáramos el problema en función de la actitud del Estado exclusivamente - lo que constituiría en realidad una simplificación inaceptable- quedarían, además de la continuidad de la situación actual, dos alternativas: evolución hacia una intervención más fuerte aún, hasta la centralización total de las economías; o vuelta del Estado a la actitud abstencionista. Objetivamente, ninguna de las dos alternativas puede ser excluída $^{80}$. La primera -que podría darse por causas políticas o sociales-

\footnotetext{
${ }^{78}$ Tales objetivos no se excluían ni en los tiempos en que el Estado, por regla general, se abstenía de intervenir en los asuntos económicos.

${ }^{79}$ En general si bien estimamos poco probable la reaparición de crisis y depresiones como las que caracterizaron la época del capitalismo en apogeo, consideramos que cualquier conclusión al respecto debe formularse con la mayor prudencia ya que, -como con mucha razón se que, durante toda esta posguerra, "el mundo de los negocios no ha sido puesto a una prueba realmente seria".

${ }^{80}$ Aunque hay síntomas para una convergencia de los sistemas. Cfr. J. TINBERGEN "Do comunist and Free Societies show a converging Pattern?" Soviet Studies (Oxford) Vol.
} 
tendría por efecto la desaparición de las fluctuaciones actuales (que todavía pueden llamarse "coyunturales", ya que la actitud del Estado se ajusta, en gran medida a la situación económica, determinada, a su ves, en gran medida por decisiones libres de las empresas, guiadas por un espíritu de lucro) las que muy probablemente serían reemplazadas por otras ${ }^{81}$ que surgirían por las condiciones creadas por el sistema respectivo. Pero tampoco hay que excluir en forma absoluta, como posible, la otra alternativa ${ }^{82}$. No hay que considerar como totalmente imposible que condiciones muy especiales (como p. ej. progresos técnicos que permitan rebajas de costos espectaculares y que hagan relegar los problemas de la ocupación y la distribución de ingresos; o una expansión espacial que haga reeditar las condiciones de la expansión de la época del capitalismo en apogeo ${ }^{83}$ tengan como consecuencia la vuelta a una actitud no intervencionista del Estado. Evidentemente, aún en tal caso, la reaparición del "ciclo económico" clásico estría ampliamente condicionada por las actitudes de los otros participantes del proceso económico así como por los demás factores tratados. Pero todo esto corresponde ya al campo de las especulaciones, en el cual no queremos aventurarnos.

Lascar Savenau Universidad Nacional del Sur

\footnotetext{
XII, No 4 (abril 1961); A. PREDOEHL, op. cit., p. 108 y ss.; J. TINBERGEN y otros, Convergencia de los sistemas económicos del Este y del Oeste y otros ensayos, Buenos Aires, 1968, Centro editor de AMÉRICA Latina.

${ }^{81}$ Como a las que se refieren los trabajos citados en la nota 77 de este trabajo.

${ }^{82} \mathrm{Si}$ bien en un sentido estricto ninguna evolución es reversible, no creemos que se pueda aplicar el criterio de "irreversible" (Cfr. CORDEBAS, op. cit., p. 105) a situaciones futuras que, aun no siendo iguales a situaciones del pasado, les sean similares en sus rasgos esenciales.

${ }^{83}$ En el sentido de PREDOEHL.
} 